\title{
Propensity Score Matching Analysis of the Effect of Payer Status on the Survival of Colon Cancer Patients
}

\author{
Lawrence Shi ${ }^{1}$, Winston Suh ${ }^{2}$, Mindie M. Kavanaugh ${ }^{2}$, Glenn Mills ${ }^{2}$, Sarah Thayer $^{3}$, Runhua Shi ${ }^{2}$ \\ 1. Hematology-Oncology, Tulane University, New Orleans, USA 2. Hematology-Oncology, Department of Medicine \& \\ Feist-Weiller Cancer Center, Louisiana State University Health Shreveport, Shreveport, USA 3. Surgical Oncology, \\ Department of Medicine \& Feist-Weiller Cancer Center, Louisiana State University Health Shreveport, Shreveport, USA
}

Corresponding author: Winston Suh,wsuh@lsuhsc.edu

\section{Abstract}

\section{Background and objective}

Colon cancer is one of the most common types of cancer globally. The factors that could affect colon cancer survival include age, stage, treatment, and other socioeconomic aspects. Payer status has been shown to be a significant predictor of cancer patient survival in retrospective studies. However, due to the limitations of retrospective studies, patient baseline characteristics between payer statuses are not comparable. Few studies have addressed the effect of payer status on the overall survival (OS) of patients using propensity score matching (PSM). In light of this, we conducted a study to examine the effect of payer status on the survival of colon cancer patients based on PSM.

\section{Materials and methods}

About 66,493 stage II/III colon cancer patients aged 40-90 years and diagnosed between 2004 and 2015 were analyzed from a de-identified National Cancer Database (NCDB) file. All patients had undergone surgery, and patients who had received radiation therapy, hormone therapy, immunotherapy, palliative care, or therapies other than chemotherapy were excluded. Only private or Medicaid payer status was included. The propensity score was calculated by computing the probability of patients being in the Medicaid group using logistic regression. The PSMATCH procedure in the SAS software (SAS Inc., Gary, NC) was used to perform PSM on patients with Medicaid and private insurance. The greedy nearest neighbor matching method was used to match one Medicaid to one privately insured patient with a caliper of 0.2 . At the same time, an exact match was done for gender, age group, race, and stage at diagnosis. Multivariate Cox regression was then used to estimate the effect of payer status on survival before and after PSM.

\section{Results}

Among the 66,493 patients, $90.3 \%$ were privately insured and $9.7 \%$ had Medicaid. In univariate analysis, payer status was found to be a significant predictor of OS. Prior to PSM, the median overall survival (MOS) for patients with private insurance was 12.75 years, while those with Medicaid had a MOS of 9.02 years. After PSM, 6,167 paired patients were matched, and patients with private insurance had a MOS of $>12.82$ years and Medicaid patients had a MOS of 8.88 years. After PSM, patients with Medicaid had a 50\% increased risk of death, and payer status proved to be a statistically significant predictor of OS of colon cancer.

\section{Conclusion}

Based on our findings, as per the PSM method, payer status can be a significant predictor of survival among colon cancer patients. Also, chemotherapy, race, age, and other socioeconomic factors were also found to be significant predictors of OS. Further research should be conducted to investigate other covariates not studied here and the mediation effect of payer on the survival of cancer patients.

Categories: Oncology, Public Health, Epidemiology/Public Health

Keywords: payer status, colon cancer, retrospective study, healthcare insurance, propensity score matching (psm)

\section{Introduction}

As healthcare reform in the United States continues to evolve, defining the impact of payer status on health outcomes remains a challenging prospect. Several studies have uncovered a link between uninsured/underinsured payer status and higher risks of mortality from cancer [1-8]. Many expect the shift in insurance coverage across the United States to continue [9-13], and the effect that this shift will have on cancer patients is still uncertain.

Colon cancer is a very common disease and accounts for a third of all cancer-related deaths in the United States among both men and women. From 2012-2016, new cases of colorectal cancer for men and women 
amounted to 38.6 per 100,000 people, and there were 14.2 deaths per 100,000 people per year. Among both genders, blacks had the highest number of new cases and deaths per 100,000 persons. The American Cancer Society estimates that there will be 149,500 new cases of colorectal cancer (104,270 colon and 45,230 rectal cancer cases) and 52,980 deaths in the United States in 2021 [14]. While the Surveillance, Epidemiology, and End Results (SEER) data from 2009-2015 has reported an overall 64.4\% five-year relative survival rate with the highest percentage of colorectal cancer deaths among people aged 75-84 [15], colorectal cancer-related deaths have been dropping for several decades due to technological and medical advances that allow for earlier detection and a higher quality of treatment, respectively [14]. As a result, there are now more than 1.5 million colon cancer survivors in the United States, and the number is continuing to rise [14]. Fortunately, the survival rate from colorectal cancer has been steadily increasing over the past 20 years, likely due to greater participation in screening, earlier diagnoses, and improvements in treatment [16,17]. However, as these advances become more widely available to those with insurance, we must also consider the impact this might have on healthcare coverage disparities.

The impact of demographic, socioeconomic, and geographic disparities on the overall survival (OS) of patients with colorectal cancer has been studied extensively [18-28]. The black population has been associated with significantly worse OS compared to other groups, due to factors such as fewer as well as lower quality screenings [19,22] or a greater likelihood to be diagnosed with late-stage cancers [23]. Regarding gender, women have been shown to have better OS compared to men [18]. Diagnoses that are more recent have been associated with better OS (likely due to advances in treatment) while older age has been linked to a greater risk of proximal tumors [18], which have ultimately been shown to have worse mortality rates [29].

Other studies have shown patients from socioeconomically deprived neighborhoods to be at greater risk of death from colorectal cancer [20,21], while a lower education acts as a barrier to proper cancer screening uptake [24]. Patients who live in less urbanized areas have been shown to refuse treatment more often, likely due to lack of access [21], and longer distances to screening and treatment centers have been associated with a greater chance to present with metastatic disease [30] or further obstruction to quality care [31]. Patients who present with comorbidities have been shown to have a higher overall risk of death [20] while chemotherapy treatment has been linked to better survival outcomes [32]. Any significant delay in treatment from the point of diagnosis can be detrimental to prognosis [21]. Even facility type has been shown to be a key factor with treatment at a community care cancer center linked to a greater risk of death [33,34].

The focus of this study is the effect of payer status on colon cancer survival. Some studies on colorectal cancer have linked data between the SEER and Center for Medicare Service to investigate the effect of these variables on survival, but not with payer status. The role of payer status in OS has only recently begun to garner attention, and recent studies have demonstrated a link between the two [6,35-37]. One study has concluded that Medicaid and uninsured patients had a $>40 \%$ higher risk of death compared to patients with private insurance [5]. Another study has revealed similar findings and shown that Medicaid and uninsured patients had worse OS compared to other patients, even after adjusting for other variables [3].

A statistically significant relationship between payer status and cancer patient survival has been demonstrated in previously published retrospective studies $[33,38]$. However, in retrospective studies, patient baseline characteristics between payer status are not comparable [32]. If payer status was shown to have an effect on the OS, such an effect could then be due to other unbalanced factors. To overcome the lack of comparability of baseline characteristics in retrospective studies, the propensity score matching (PSM) method can be used to balance patient characteristics between payer status groups [32,39]. Thus, this study has employed the PSM method to assess the effect of payer status on the survival of colon cancer patients.

\section{Materials And Methods}

This study examined 66,493 patients with stage II or III colon cancer who were registered between 2004 and 2015 and followed up until December 31, 2015. The data was derived from a de-identified National Cancer Database (NCDB) file. The NCDB documents approximately $70 \%$ of all newly diagnosed cases of cancer in the United States at the institutional level [31]. Codes from the International Classification of Disease for Oncology, third edition (ICD-O-3) associated with a diagnosis of colon cancer (C180-189) were used to select patients. All patients had undergone surgery, and patients who had received radiation therapy, hormone therapy, immunotherapy, palliative care, or therapies other than chemotherapy were excluded. Only patients with private insurance or Medicaid were included, while those with unknown insurance, Medicare, and other insurance types were excluded.

The survival duration of colon cancer patients was calculated from the date of diagnosis to the date of death, date of loss to follow-up, or date of study conclusion (December 31, 2015). The variables investigated included payer status, sex, age, race, Charlson Comorbidity score, income, education, distance traveled, facility type, diagnosing/treating facility, treatment delay, stage, year of diagnosis, and chemotherapy.

The patients were classified into two groups based on age: those aged 40-64 years and those of 65+ years. Patient race was categorized as white or black. Charlson Comorbidity [32] is an index that characterizes the overall health status of a patient. Charlson Comorbidity was categorized as "yes" or "no" to indicate 
whether a patient did or did not present with a comorbidity along with initial cancer diagnosis. Zip Code level income and education were also used as variables in this research. Patients were further classified into different groups based on income (household income of $<36,000$ or $>36,000$ USD per year) as well as education ( $>20 \%$ to denote at least a high school education while $<20 \%$ denoted those who did not receive a high school education). Distance traveled to the facility was defined as $<30$ or $>30$ miles and the facility type was determined based on the NCDB's categorization of Comprehensive Community Cancer and Academic/Research program. Diagnosing/treating facility was categorized based on whether patients were diagnosed and treated at the same initial facility or a different facility. Treatment delay from diagnosis was grouped as 0-30 or >31 days; tumor stage was defined based on the American Joint Committee on Cancer's (AJCC) categorization of stage II and III tumors; classification based on years of diagnosis included patients diagnosed between 2004-2009 and those between 2010-2014. The treatment status was categorized as either "received" or "not received" for chemotherapy.

The propensity score was calculated by computing the probability of patients being in the Medicaid group using logistic regression. The PSMATCH procedure in the SAS software (SAS Inc., Gary, NC) was used to perform PSM [30] on patients with Medicaid and private insurance. The greedy nearest neighbor matching method [39] was used to match one Medicaid to one privately insured patient with a caliper of 0.2 . At the same time, an exact match was done for gender, age group, race, and stage at diagnosis. A caliper measure such as "caliper $=0.5$ " is the caliper requirement used for matching, meaning that the difference in PS logits must be less than or equal to 0.5 times the estimated logit of the standard deviation.

Patient characteristics were compared between private and Medicaid patients before and after matching by using the chi-square test. The Kaplan-Meier/product-limit method was used to estimate the survival of the patients. The log-rank test was used to compare the OS for payer status. Multivariate Cox regression was used to simultaneously estimate the hazard of death [hazard ratio (HR)] for payer status and adjust for other factors. The statistical software SAS 9.4 was used for data management, statistical analysis, and modeling. All p-values $<0.05$ or $95 \%$ confidence intervals (CI) not including one were considered statistically significant.

\section{Results}

Of the 66,493 stage II and stage III colon cancer patients included in this study, $90.30 \%$ had private insurance and $9.70 \%$ had Medicaid prior to the PSM. Of the 6,167 pairs of patients included after PSM, 50\% had private insurance and 50\% had Medicaid. The demographic and clinical characteristics of the patient population prior to and after PSM are summarized in Table 1. Before PSM, excluding stage and distance traveled, there was a statistically significant difference between private insurance and Medicaid patients in terms of gender, race, age, year of diagnosis, education, income, comorbidity, education, facility type, diagnosing/treating facility, treatment delay, and chemotherapy ( $\mathrm{p}<0.0001$ ). For example, before PSM, $22.2 \%$ and $30.2 \%$ of colon cancer patients under private insurance and Medicaid, respectively, had comorbidities, and this was determined to be a significant factor in colon cancer survival.

However, after PSM with exact match variables on gender, age, race, and stage, there was no statistically significant difference when comparing between private insurance and Medicaid for all other tested variables. For example, after PSM, 30.36\% and 30.39\% of colon cancer patients under private insurance and Medicaid, respectively, had comorbidities, and these values were not statistically significant $(\mathrm{p}=0.9844)$. The same process can be applied to all variables to conclude that the final group of private and Medicaid patients had, among other variables, a similar demographic and socioeconomic status, clinical characteristics, and treatment and that any difference in analyzed survival should now be reliant on payer status. 


\section{Cureus}

\begin{tabular}{|c|c|c|c|c|c|c|c|c|c|c|c|}
\hline \multirow[t]{3}{*}{ Characteristics } & \multirow[t]{3}{*}{ Subtypes } & \multicolumn{5}{|c|}{ Prior to $\operatorname{PSM}(n=60,042,6,451)$} & \multicolumn{5}{|c|}{ After PSM $(n=6,167,6,167)$} \\
\hline & & \multicolumn{2}{|l|}{ Private } & \multicolumn{2}{|c|}{ Medicaid } & \multirow[t]{2}{*}{ P-value } & \multicolumn{2}{|c|}{ Private } & \multicolumn{2}{|c|}{ Medicaid } & \multirow[t]{2}{*}{ P-value } \\
\hline & & $\mathrm{N}$ & $\%$ & $\mathrm{~N}$ & $\%$ & & $\mathrm{~N}$ & $\%$ & $\mathrm{~N}$ & $\%$ & \\
\hline \multirow{2}{*}{ Gender } & Male & 30,927 & 51.51 & 3,064 & 47.50 & \multirow{2}{*}{$<0.0001$} & 2,924 & 47.41 & 2,924 & 47.41 & \multirow{2}{*}{1.0000} \\
\hline & Female & 29,115 & 48.49 & 3,387 & 52.50 & & 3,243 & 52.59 & 3,243 & 52.59 & \\
\hline \multirow{2}{*}{ Race } & White & 52,420 & 87.31 & 4,616 & 71.55 & \multirow{2}{*}{$<0.0001$} & 4,401 & 71.36 & 4,401 & 71.36 & \multirow{2}{*}{1.0000} \\
\hline & Black & 7,622 & 12.69 & 1,835 & 28.45 & & 1,766 & 28.64 & 1,766 & 28.64 & \\
\hline \multirow{2}{*}{ Age (years) } & $40-64$ & 45,657 & 76.04 & 5,181 & 80.31 & \multirow{2}{*}{$<0.0001$} & 2,924 & 47.41 & 2,924 & 47.41 & \multirow{2}{*}{1.0000} \\
\hline & $\geq 65$ & 14,385 & 23.96 & 1,270 & 19.69 & & 3,243 & 52.59 & 3,243 & 52.59 & \\
\hline \multirow{2}{*}{ Year of diagnosis } & 2004-2009 & 32,691 & 54.45 & 2,891 & 44.81 & \multirow{2}{*}{$<0.0001$} & 2,746 & 44.53 & 2,750 & 44.59 & \multirow{2}{*}{0.9567} \\
\hline & 2010-2014 & 27,351 & 45.55 & 3,560 & 55.19 & & 3,421 & 55.47 & 3,417 & 55.41 & \\
\hline \multirow{2}{*}{ Distance traveled (miles) } & $<30$ & 50,617 & 85.21 & 5,443 & 85.11 & \multirow{2}{*}{0.8385} & 5,293 & 85.82 & 3,064 & 49.77 & \multirow{2}{*}{0.2302} \\
\hline & $\geq 30$ & 8,788 & 14.79 & 952 & 14.89 & & 874 & 14.18 & 3,103 & 51.34 & \\
\hline \multirow{2}{*}{ Income (USD thousand) } & $<36$ & 16,310 & 28.12 & 3,179 & 50.48 & \multirow{2}{*}{$<0.0001$} & 3,118 & 50.56 & 3,111 & 50.45 & \multirow{2}{*}{0.9140} \\
\hline & $\geq 36$ & 41,685 & 71.88 & 3,118 & 49.52 & & 3,049 & 49.44 & 3,056 & 49.55 & \\
\hline \multirow{2}{*}{ Comorbidity } & No & 46,708 & 77.79 & 4,503 & 69.80 & & 4,295 & 69.64 & 4,293 & 69.61 & \\
\hline & Yes & 13,334 & 22.21 & 1,948 & 30.20 & & 1,872 & 30.36 & 1,874 & 30.39 & \\
\hline & $\geq 20 \%$ & 21,702 & 37.42 & 3,810 & 60.51 & & 3,753 & 60.86 & 3,720 & 60.32 & \\
\hline & $<20 \%$ & 36,287 & 62.58 & 2,487 & 39.49 & & 2,414 & 39.14 & 2,447 & 39.68 & \\
\hline & СССР & 38,289 & 63.77 & 3,280 & 50.84 & & 3,180 & 51.56 & 3,140 & 50.92 & \\
\hline & Academic/research & 21,753 & 36.23 & 3,171 & 49.16 & & 2,987 & 48.44 & 3,027 & 49.08 & \\
\hline & Same facility & 37,272 & 62.08 & 4,313 & 66.86 & & 4,173 & 67.67 & 4,190 & 67.94 & \\
\hline & Different facility & 22,770 & 37.92 & 2,138 & 33.14 & & 1,994 & 32.33 & 1,977 & 32.06 & \\
\hline & $0-30$ & 49,830 & 84.86 & 5,193 & 81.95 & & 5,107 & 82.81 & 5,060 & 82.05 & \\
\hline & $\geq 31$ & 8,893 & 15.14 & 1,144 & 18.05 & & 1,060 & 17.19 & 1,107 & 17.95 & \\
\hline & II & 27,128 & 45.18 & 2,898 & 44.92 & & 2,777 & 45.03 & 2,777 & 45.03 & \\
\hline & III & 32,914 & 54.82 & 3,553 & 55.08 & & 3,390 & 54.97 & 3,390 & 54.97 & \\
\hline & No & 21,955 & 36.57 & 2,494 & 38.66 & & 2,349 & 38.09 & 2,389 & 38.74 & \\
\hline & Yes & 38,087 & 63.43 & 3,957 & 61.34 & & 3,818 & 61.91 & 3,778 & 61.26 & \\
\hline
\end{tabular}

TABLE 1: Demographics and clinical characteristics of patients with private and Medicaid insurance before and after PSM

CCCP: Comprehensive Community Cancer Program; AJCC: American Joint Committee on Cancer; PSM: propensity score matching

In univariate analysis, OS according to payer status before and after PSM is presented in Figure 1 and Figure 2. Figure 1 shows that prior to PSM, the median overall survival (MOS) was approximately 9.02 years for Medicaid patients, while patients with private insurance had a MOS of 12.75 years. 


\section{Cureus}

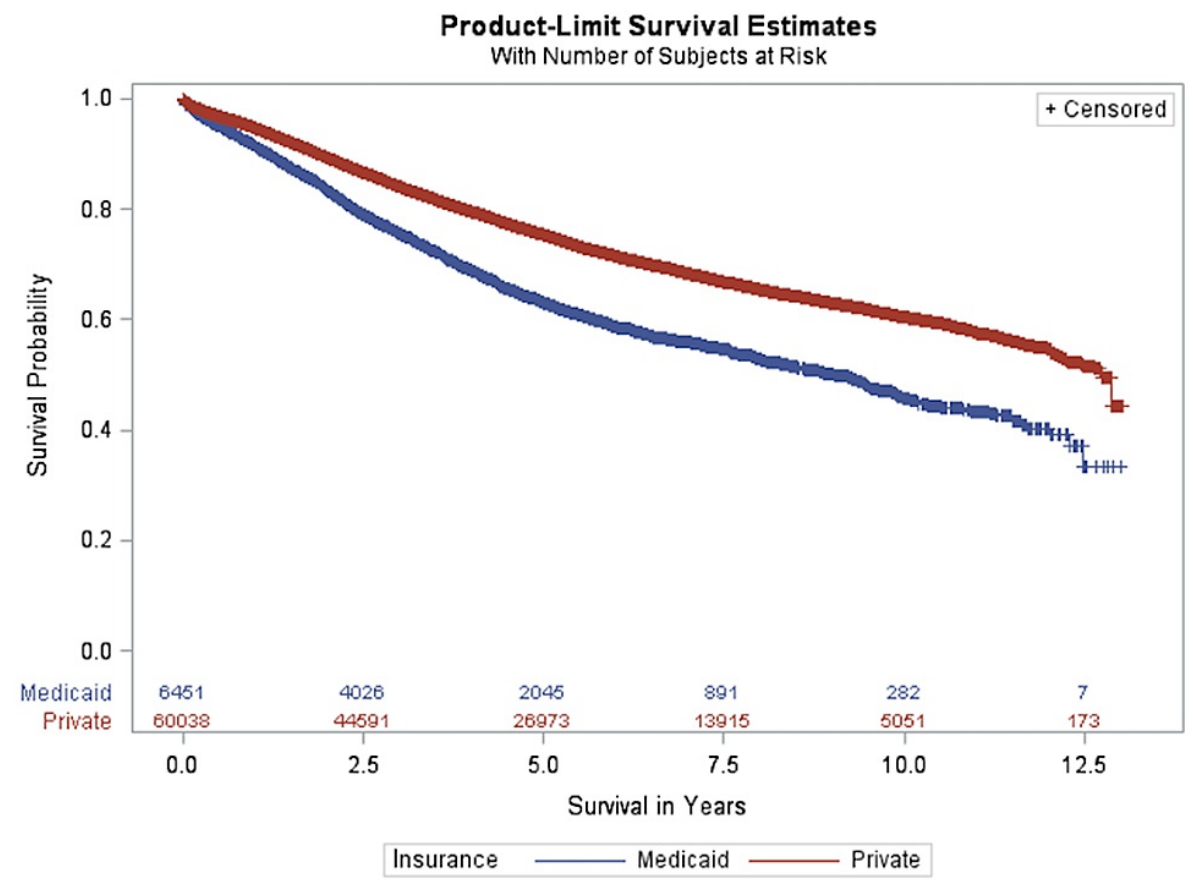

FIGURE 1: Overall survival according to payer status prior to propensity score matching

Figure 2 illustrates the OS by payer status after PSM. The MOS was approximately 8.88 years for patients with Medicaid, while patients with private insurance had a MOS of more than 12.82 years. Thus, even with matching patient characteristics, payer status was found to play a role in the survival of the patients.

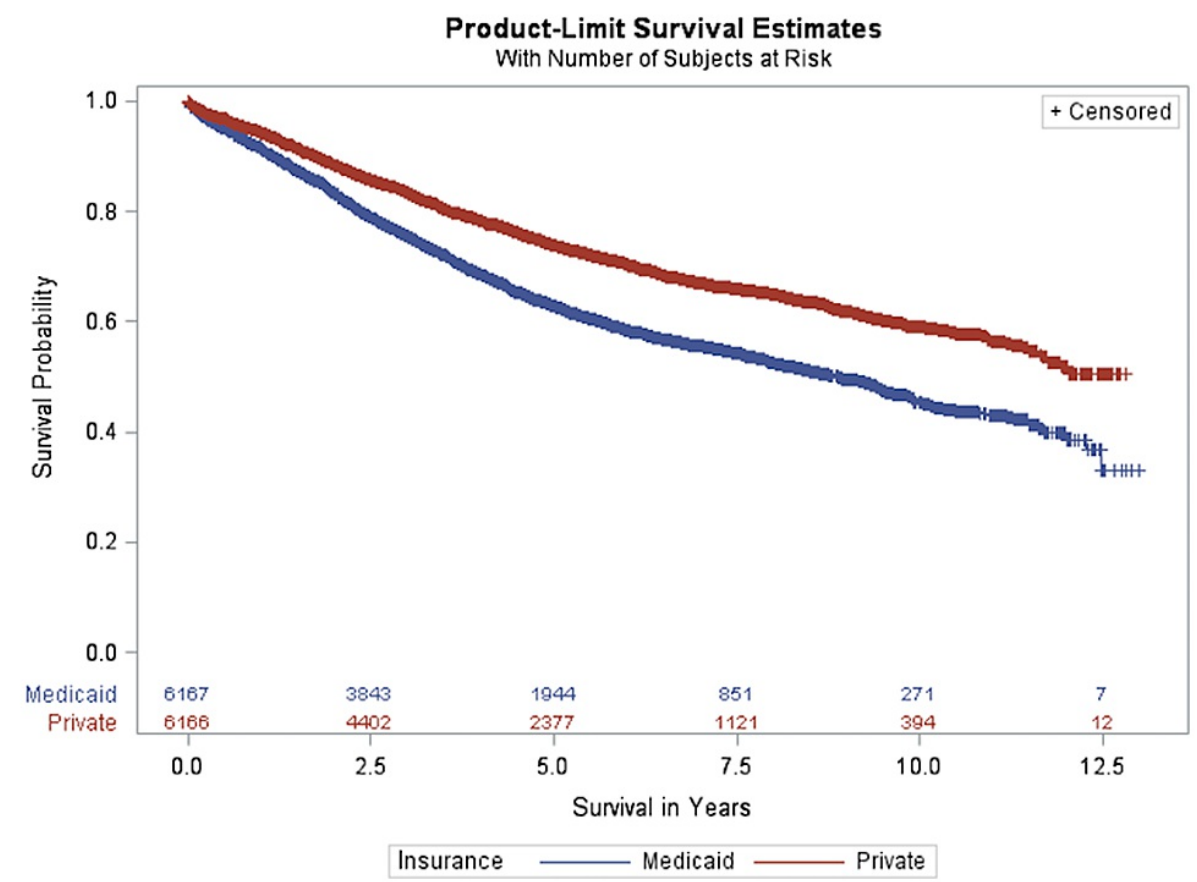

FIGURE 2: Overall survival according to payer status after propensity score matching

Table 2 displays the HR and the $95 \% \mathrm{CI}$ of $\mathrm{HR}$ for each variable from multivariate Cox regression analysis prior to and after PSM. Payer status was a significant predictor of OS ( $\mathrm{p}<0.0001)$ after controlling for gender, 


\section{Cureus}

race, age, year of diagnosis, distance traveled, income, comorbidity, education, facility type, diagnosing/treating facility, treatment delay, stage, and chemotherapy before PSM.

In multivariate Cox regression analysis, after adjusting for other factors, the HR prior to PSM for Medicaid patients was 1.54 compared to patients with private insurance. In other words, when compared to private insurance patients, patients with Medicaid were $54 \%$ more likely to die. After PSM, the HR was 1.50 for patients with Medicaid compared to patients with private insurance. Similar results were found after adjusting for factors including gender, race, age, year of diagnosis, distance traveled, income, comorbidity, education, facility type, diagnosing/treating facility, treatment delay, stage, and chemotherapy.

After PSM, a majority of factors including race, year of diagnosis, distance traveled, income, education, facility type, diagnosing/treating facility, and treatment delay were found to be significant predictors of OS. 


\section{Cureus}

\begin{tabular}{|c|c|c|c|c|c|c|c|c|c|}
\hline \multirow[t]{3}{*}{ Characteristics } & \multirow[t]{3}{*}{ Subtypes } & \multicolumn{4}{|c|}{ Prior to PSIM } & \multicolumn{4}{|c|}{ After PSIM } \\
\hline & & \multirow[t]{2}{*}{ HR } & \multicolumn{2}{|l|}{$95 \% \mathrm{Cl}$} & \multirow[t]{2}{*}{ P-value } & \multirow[t]{2}{*}{ HR } & \multicolumn{2}{|l|}{$95 \% \mathrm{Cl}$} & \multirow[t]{2}{*}{ P-value } \\
\hline & & & Lower & Upper & & & Lower & Upper & \\
\hline \multirow{2}{*}{ Insurance } & Private & 1.00 & & & & & & & \\
\hline & Medicaid & 1.54 & 1.47 & 1.62 & $<0.0001$ & 1.50 & 1.41 & 1.60 & $<0.0001$ \\
\hline \multirow{2}{*}{ Gender } & Female & 1.00 & & & & & & & \\
\hline & Male & 1.17 & 1.14 & 1.21 & $<0.0001$ & 1.22 & 1.14 & 1.30 & $<0.0001$ \\
\hline \multirow{2}{*}{ Race } & White & 1.00 & & & & & & & \\
\hline & Black & 1.14 & 1.09 & 1.19 & $<0.0001$ & 1.08 & 1.01 & 1.16 & 0.0354 \\
\hline \multirow{2}{*}{ Age (years) } & 40-64 & 1.00 & & & & & & & \\
\hline & $\geq 65$ & 2.24 & 2.17 & 2.32 & $<0.0001$ & 1.70 & 1.58 & 1.83 & $<0.0001$ \\
\hline \multirow{2}{*}{ Year of diagnosis } & 2010-2014 & 1.00 & & & & & & & \\
\hline & $2004-2009$ & 1.06 & 1.03 & 1.10 & 0.0005 & 1.12 & 1.05 & 1.21 & 0.0014 \\
\hline \multirow{2}{*}{ Distance traveled (miles) } & $<30$ & 1.00 & & & & & & & \\
\hline & $\geq 30$ & 1.08 & 1.03 & 1.13 & 0.001 & 1.11 & 1.02 & 1.22 & 0.0236 \\
\hline \multirow{2}{*}{ Income (USD thousand) } & $\geq 36$ & 1.00 & & & & & & & \\
\hline & $<36$ & 1.10 & 1.05 & 1.14 & 0 & 1.13 & 1.05 & 1.22 & 0.0017 \\
\hline \multirow{2}{*}{ Comorbidity } & No & 1.00 & & & & & & & \\
\hline & Yes & 1.43 & 1.39 & 1.48 & $<0.0001$ & 1.38 & 1.29 & 1.47 & $<0.0001$ \\
\hline \multirow{2}{*}{ Education } & $\geq 20 \%$ & 1.00 & & & & & & & \\
\hline & $<20 \%$ & 0.99 & 0.96 & 1.03 & 0.7354 & 1.01 & 0.93 & 1.09 & 0.8461 \\
\hline \multirow{2}{*}{ Facility type } & Academic/research & 1.00 & & & & & & & \\
\hline & СССР & 1.06 & 1.03 & 1.10 & 0.0001 & 1.08 & 1.01 & 1.15 & 0.0204 \\
\hline \multirow{2}{*}{ Diagnosing/treating facility } & Different facility & 1.00 & & & & & & & \\
\hline & Same facility & 1.19 & 1.15 & 1.24 & $<0.0001$ & 1.10 & 1.02 & 1.18 & 0.0186 \\
\hline \multirow{2}{*}{ Treatment delay (days) } & $\geq 31$ & 1.00 & & & & & & & \\
\hline & $0-30$ & 1.10 & 1.05 & 1.15 & $<0.0001$ & 1.18 & 1.08 & 1.30 & 0.0003 \\
\hline \multirow{2}{*}{ AJCC stage } & II & 1.00 & & & & & & & \\
\hline & III & 2.29 & 2.20 & 2.38 & $<0.0001$ & 2.15 & 1.98 & 2.33 & $<0.0001$ \\
\hline \multirow{2}{*}{ Chemotherapy } & Yes & 1.00 & & & & & & & \\
\hline & No & 1.83 & 1.76 & 1.90 & $<0.0001$ & 1.91 & 1.76 & 2.07 & $<0.0001$ \\
\hline
\end{tabular}

\section{TABLE 2: Multivariate analysis of predictors of overall survival prior to and after PSM}

CCCP: Comprehensive Community Cancer Program; AJCC: American Joint Committee on Cancer; PSM: propensity score matching; HR: hazard ratio; Cl: confidence interval

\section{Discussion}

This study effectively demonstrates that payer status has a statistically significant effect on the OS of colon cancer patients after adjusting for all other predictive factors through the PSM method. Medicaid patients had a $50 \%$ increased risk of death compared to those with private insurance. Our findings are consistent with previous studies on payer status and cancer patient survival $[1,3,5-7,36]$. Although the mechanism by which 
payer status affects OS is not entirely clear, it could be mediated, among numerous other variables, through differences in access to certain treatment types [40], proper screenings [41], or improper follow-up following a diagnosis [1]. Further research may be warranted to investigate this observation through mediation analysis.

Similar to findings from other studies, we observed that patients with higher incomes and higher education had better survival from cancer [30,42,43]. In addition, our data agree with other studies that show that male gender, advanced age, African American race, and a higher Charlson Comorbidity score are associated with decreased OS $[18,27,44,45]$. Our findings are also in line with the results of previous studies regarding the effect of tumor stage and treatment [43,46], distance traveled [30], and year of diagnosis [18] on patient survival. Finally, other factors (facility type, treatment delay, and diagnosing/treating facility) also showed an association with OS, consistent with findings in the current literature [47]. All the above predictive factors were adjusted for in our final analysis while assessing the effect of payer status on patient OS. Overall, we determined that payer status has a statistically significant relationship with OS.

Prior to PSM, it was discovered that patient demographics, socioeconomic status, and clinical characteristic were significantly different between private and Medicaid patient groups (Table 1). Differences in survival between private insurance patients and Medicaid patients were already found before PSM (Figure 1). Thus, the difference found in survival may not be completely due to payer status but could also be due to differences in other factors such as gender, race, age, and comorbidities. For example, prior to PSM, 22.2\% and $30.2 \%$ of colon cancer patients under private insurance and Medicaid, respectively, had comorbidities. Other studies have indicated that the presence of more comorbidities leads to a shorter survival [39]. All other factors except distance traveled and stage have been shown to have a significant effect on colon cancer survival including male gender [18], black race [19,22], and old age [18], which have all been linked to a worse survival rate.

After PSM, the differences in patient characteristics and clinical status listed above were not found to be statistically significant between private insurance and Medicaid. For example, after PSM, 30.36\% and 30.39\% of patients under private insurance and Medicaid, respectively, had comorbidities $(\mathrm{p}=0.9844)$. Other factors like gender, race, age, and stage were exact matches and had p-values of 1.000 . Therefore, it was determined that the difference in survival between private insurance and Medicaid colon cancer patients (Figure 2) is due to the difference in payer status alone.

In multivariate Cox regression analysis with PSM, it was shown that patients with Medicaid were $50 \%$ more likely to die compared to private insurance patients. In addition, we demonstrated that patients who do not receive chemotherapy are $91 \%$ more likely to die compared to patients who receive chemotherapy. After PSM in multivariate Cox regression analysis, covariates such as gender, race, age, year of diagnosis, distance traveled, income, comorbidity, education, facility type, diagnosing/treating facility, treatment delay, and stage were controlled for and payer status was found to be a significant factor in the survival of colon cancer patients.

Despite the efforts to account for as many confounding variables as possible while utilizing a large sample population, there are still some limitations to this study. Due to the limited number of variables that we could apply in our analysis, there may still be a few important confounding variables that we could not control for. Furthermore, this study only investigated stage II and stage III colon cancer patients who had undergone surgery and chemotherapy as treatment. Hence, the results from this study may not apply to other patient populations. Data on Education and income were collected by ZIP Code rather than by patient or household. Utilizing individual or household income in the analysis would have strengthened the results. Information regarding specific causes of death was also not available in the NCDB file. Measuring the effect of payer status on cause-specific survival might yield different results.

\section{Conclusions}

This study has indicated that after PSM, patient demographics, socioeconomic status, and clinical characteristics were similar between private insurance and Medicaid patients. However, the survival differences between these two groups still remained after PSM, and it was determined that it could be caused by the effect of the payer status. Payer status was found to play a role in the survival of colon cancer patients even after adjusting for other variables. These timely findings shed light on the significant impact of payer status on the overall survival of colon cancer patients. As we continue to navigate our way through the dynamically changing landscape of healthcare reform in the United States today, it is important to consider the influence of payer status on health outcomes in the future.

\section{Additional Information}

\section{Disclosures}

Human subjects: All authors have confirmed that this study did not involve human participants or tissue. Animal subjects: All authors have confirmed that this study did not involve animal subjects or tissue. Conflicts of interest: In compliance with the ICMJE uniform disclosure form, all authors declare the 
following: Payment/services info: All authors have declared that no financial support was received from any organization for the submitted work. Financial relationships: All authors have declared that they have no financial relationships at present or within the previous three years with any organizations that might have an interest in the submitted work. Other relationships: All authors have declared that there are no other relationships or activities that could appear to have influenced the submitted work.

\section{Acknowledgements}

The authors wish to acknowledge the Commission on Cancer of the American College of Surgeons and the American Cancer Society for making public data available through the NCDB. The data used in this study were derived from a de-identified NCDB file. The American College of Surgeons and the Commission on Cancer have not verified and are not responsible for the analytic or statistical methodology employed or the conclusions drawn from these data by the investigator.

\section{References}

1. Ward E, Halpern M, Schrag N, et al.: Association of insurance with cancer care utilization and outcomes . CA Cancer J Clin. 2008, 58:9-31. 10.3322/CA.2007.0011

2. Yim J, Hwang SS, Yoo KY, Kim CY: Contribution of income-related inequality and healthcare utilisation to survival in cancers of the lung, liver, stomach and colon. J Epidemiol Community Health. 2012, 66:37-40. 10.1136/jech.2009.104554

3. Parikh AA, Robinson J, Zaydfudim VM, Penson D, Whiteside MA: The effect of health insurance status on the treatment and outcomes of patients with colorectal cancer. J Surg Oncol. 2014, 110:227-32. $10.1002 /$ jso.23627

4. Ortiz-Ortiz KJ, Ramírez-García R, Cruz-Correa M, Ríos-González MY, Ortiz AP: Effects of type of health insurance coverage on colorectal cancer survival in Puerto Rico: a population-based study. PLoS One. 2014, 9:e96746. 10.1371/journal.pone.0096746

5. Roetzheim RG, Pal N, Gonzalez EC, Ferrante JM, Van Durme DJ, Krischer JP: Effects of health insurance and race on colorectal cancer treatments and outcomes. Am J Public Health. 2000, 90:1746-54. 10.2105/ajph.90.11.1746

6. Shi R, Mills G, McLarty J, Burton G, Shi Z, Glass J: Commercial insurance triples chances of breast cancer survival in a public hospital. Breast J. 2013, 19:664-7. 10.1111/tbj.12185

7. Shi R, Taylor H, McLarty J, Liu L, Mills G, Burton G: Effects of payer status on breast cancer survival: a retrospective study. BMC Cancer. 2015, 15:211. 10.1186/s12885-015-1228-7

8. Wan N, Zhan FB, Lu Y, Tiefenbacher JP: Access to healthcare and disparities in colorectal cancer survival in Texas. Health Place. 2012, 18:321-9. 10.1016/j.healthplace.2011.10.007

9. Albright HW, Moreno M, Feeley TW, Walters R, Samuels M, Pereira A, Burke TW: The implications of the 2010 Patient Protection and Affordable Care Act and the Health Care and Education Reconciliation Act on cancer care delivery. Cancer. 2011, 117:1564-74. 10.1002/cncr.25725

10. Brawley OW, Virgo KS: From the guest editors: introduction for the impact of health care reform on cancer patients. Cancer J. 2010, 16:551-3. 10.1097/PPO.0b013e3182031591

11. Ferris LW, Farber M, Guidi TU, Laffey WJ: Impact of health care reform on the cancer patient: a view from cancer executives. Cancer J. 2010, 16:600-5. 10.1097/PPO.0b013e3181feeba1

12. Schwartz K, Claxton G: The Patient Protection and Affordable Care Act: how will it affect private health insurance for cancer patients?. Cancer J. 2010, 16:572-6. 10.1097/PPO.0b013e3181ff292a

13. Virgo KS, Burkhardt EA, Cokkinides VE, Ward EM: Impact of health care reform legislation on uninsured and Medicaid-insured cancer patients. Cancer J. 2010, 16:577-83. 10.1097/PPO.0b013e31820189cb

14. What are the key statistics about colorectal cancer?. (2021). Accessed: June 17, 2021: https://www.cancer.org/cancer/colon-rectal-cancer/about/key-statistics.html.

15. SEER cancer stat facts: colorectal cancer. (2021). Accessed: June 17, 2021 : https://seer.cancer.gov/statfacts/html/colorect.html.

16. Colorectal cancer. (2021). Accessed: June 17, 2021: http://www.cancer.org/cancer/colonandrectumcancer/detailedguide/index.

17. Edwards BK, Ward E, Kohler BA, et al.: Annual report to the nation on the status of cancer, 1975-2006, featuring colorectal cancer trends and impact of interventions (risk factors, screening, and treatment) to reduce future rates. Cancer. 2010, 116:544-73. 10.1002/cncr.24760

18. Hendifar A, Yang D, Lenz F, et al.: Gender disparities in metastatic colorectal cancer survival . Clin Cancer Res. 2009, 15:6391-7. 10.1158/1078-0432.CCR-09-0877

19. Lansdorp-Vogelaar I, Kuntz KM, Knudsen AB, van Ballegooijen M, Zauber AG, Jemal A: Contribution of screening and survival differences to racial disparities in colorectal cancer rates. Cancer Epidemiol Biomarkers Prev. 2012, 21:728-36. 10.1158/1055-9965.EPI-12-0023

20. Lian M, Schootman M, Doubeni CA, et al.: Geographic variation in colorectal cancer survival and the role of small-area socioeconomic deprivation: a multilevel survival analysis of the NIH-AARP Diet and Health Study Cohort. Am J Epidemiol. 2011, 174:828-38. 10.1093/aje/kwr162

21. Liu CY, Chen WT, Kung PT, Chiu CF, Wang YH, Shieh SH, Tsai WC: Characteristics, survival, and related factors of newly diagnosed colorectal cancer patients refusing cancer treatments under a universal health insurance program. BMC Cancer. 2014, 14:446. 10.1186/1471-2407-14-446

22. Wallace K, Hill EG, Lewin DN, et al.: Racial disparities in advanced-stage colorectal cancer survival . Cancer Causes Control. 2013, 24:463-71. 10.1007/s10552-012-0133-5

23. Wassira LN, Pinheiro PS, Symanowski J, Hansen A: Racial-ethnic colorectal cancer survival disparities in the mountain west region: the case of Blacks compared to Whites. Ethn Dis. 2013, 23:103-9.

24. Tammana VS, Laiyemo AO: Colorectal cancer disparities: issues, controversies and solutions . World J Gastroenterol. 2014, 20:869-76. 10.3748/wig.v20.i4.869 
25. Ayanian JZ: Racial disparities in outcomes of colorectal cancer screening: biology or barriers to optimal care?. J Natl Cancer Inst. 2010, 102:511-3. 10.1093/jnci/djq089

26. Gill AA, Enewold L, Zahm SH, Shriver CD, Stojadinovic A, McGlynn KA, Zhu K: Colon cancer treatment: are there racial disparities in an equal-access healthcare system?. Dis Colon Rectum. 2014, 57:1059-65. 10.1097/DCR.0000000000000177

27. Fitzgerald TL, Lea CS, Brinkley J, Zervos EE: Colorectal cancer outcome inequalities: association between population density, race, and socioeconomic status. Rural Remote Health. 2014, 14:2668.

28. Aizer AA, Wilhite TJ, Chen MH, et al.: Lack of reduction in racial disparities in cancer-specific mortality over a 20-year period. Cancer. 2014, 120:1532-9. 10.1002/cncr.28617

29. Wong R: Proximal tumors are associated with greater mortality in colon cancer . J Gen Intern Med. 2010, 25:1157-63. 10.1007/s11606-010-1460-4

30. Massarweh NN, Chiang YJ, Xing Y, et al.: Association between travel distance and metastatic disease at diagnosis among patients with colon cancer. J Clin Oncol. 2014, 32:942-8. 10.1200/JCO.2013.52.3845

31. Stitzenberg KB, Sigurdson ER, Egleston BL, Starkey RB, Meropol NJ: Centralization of cancer surgery: implications for patient access to optimal care. J Clin Oncol. 2009, 27:4671-8. 10.1200/JCO.2008.20.1715

32. Kobayashi H, Kotake K, Sugihara K: Impact of adjuvant chemotherapy in patients with curatively resected stage IV colorectal cancer. Medicine (Baltimore). 2015, 94:e696. 10.1097/MD.0000000000000696

33. Master S, Munker R, Shi Z, Mills G, Shi R: Insurance status and other non-biological factors predict outcomes in acute myelogenous leukemia: analysis of data from the National Cancer Database. Anticancer Res. 2016, 36:4915-21. 10.21873/anticanres.11057

34. Shi Z, Peddi P, Burton G, Mills G, Shi R: Effect of postmastectomy radiation on survival of AJCC pN2/N3 breast cancer patients. Anticancer Res. 2016, 36:261-9.

35. Mehta V, Shi Z, Mills GM, Nathan CA, Shi R: Effect of payer status on relative survival of patients with laryngeal cancer. Anticancer Res. 2016, 36:327-33.

36. Slatore CG, Au DH, Gould MK: An official American Thoracic Society systematic review: insurance status and disparities in lung cancer practices and outcomes. Am J Respir Crit Care Med. 2010, 182:1195-205. 10.1164/rccm.2009-038ST

37. Tawk R, Abner A, Ashford A, Brown CP: Differences in colorectal cancer outcomes by race and insurance. Int J Environ Res Public Health. 2015, 13:ijerph13010048. 10.3390/ijerph13010048

38. Shi R, Taylor H, Liu L, Mills G, Burton G: Private payer's status improves male breast cancer survival . Breast J. 2016, 22:101-4. 10.1111/tbj.12523

39. Austin PC: The performance of different propensity score methods for estimating marginal hazard ratios . Stat Med. 2013, 32:2837-49. 10.1002/sim.5705

40. Rochon J, du Bois A, Lange T: Mediation analysis of the relationship between institutional research activity and patient survival. BMC Med Res Methodol. 2014, 14:9. 10.1186/1471-2288-14-9

41. Kwok J, Langevin SM, Argiris A, Grandis JR, Gooding WE, Taioli E: The impact of health insurance status on the survival of patients with head and neck cancer. Cancer. 2010, 116:476-85. 10.1002/cncr.24774

42. McLaughlin JM, Anderson RT, Ferketich AK, Seiber EE, Balkrishnan R, Paskett ED: Effect on survival of longer intervals between confirmed diagnosis and treatment initiation among low-income women with breast cancer. J Clin Oncol. 2012, 30:4493-500. 10.1200/JCO.2012.39.7695

43. Klein J, Ji M, Rea NK, Stoodt G: Differences in male breast cancer stage, tumor size at diagnosis, and survival rate between metropolitan and nonmetropolitan regions. Am J Mens Health. 2011, 5:430-7. $10.1177 / 1557988311400403$

44. SEER cancer stat facts: colon and rectum cancer. (2021). Accessed: June 17, 2021: http://seer.cancer.gov/statfacts/html/colorect.html.

45. Edwards BK, Noone AM, Mariotto AB, et al.: Annual Report to the Nation on the status of cancer, 19752010, featuring prevalence of comorbidity and impact on survival among persons with lung, colorectal, breast, or prostate cancer. Cancer. 2014, 120:1290-314. 10.1002/cncr.28509

46. Poulin-Costello M, Azoulay L, Van Cutsem E, Peeters M, Siena S, Wolf M: An analysis of the treatment effect of panitumumab on overall survival from a phase 3, randomized, controlled, multicenter trial (20020408) in patients with chemotherapy refractory metastatic colorectal cancer. Target Oncol. 2013, 8:127-36. 10.1007/s11523-013-0271-z

47. Amri R, Bordeianou LG, Sylla P, Berger DL: Treatment delay in surgically-treated colon cancer: does it affect outcomes?. Ann Surg Oncol. 2014, 21:3909-16. 10.1245/s10434-014-3800-9 